\title{
Rheological properties of suspensions of thermo-responsive poly( $N$-isopropylacrylamide) microgels undergoing volume phase transition
}

\begin{abstract}
Saori Minami ${ }^{1}$, Takumi Watanabe ${ }^{2}$, Daisuke Suzuki ${ }^{2,3}$ and Kenji Urayama ${ }^{1}$
The rheological properties of suspensions of thermo-responsive poly( $\mathrm{N}$-isopropylacrylamide) (PNIPAM) microgels are investigated in the swollen and collapsed states using oscillatory and long-time creep measurements. In the swollen state with repulsive interparticle interaction, the zero shear viscosity and quasi-plateau modulus are solely governed by the effective volume fraction $\left(\phi_{\text {eff }}\right)$ and are independent of temperature, particle diameter and particle concentration. The long-time creep tests reveal that in the collapsed state with attractive interparticle interactions, the moderately concentrated suspensions with $\phi_{\text {eff }} \approx 0.5$ become viscoelastic liquids with high viscosities of $>10^{5}$ Pas and long-terminal relaxation times of $>10^{3} \mathrm{~s}$ due to the formation of a network-like aggregation of particles, whereas the suspensions with the same $\phi_{\text {eff }}$ in the swollen state exhibit a Newtonian flow with low viscosities of less than $10^{-1}$ Pas. The network-like aggregation of particles is so fragile that it can be broken by a very small stress of several Pascals. The modulus and viscosity in the collapsed state depend on both $\phi_{\text {eff }}$ and temperature: the modulus and viscosity increase, as the temperature increases due to an increase in the hydrophobic nature of the PNIPAM chains, which govern the attractive interparticle interaction. The particle aggregations in the collapsed state considerably lower the threshold value of $\phi_{\text {eff }}\left(\phi_{\mathrm{c}}\right)$, above which the infinite percolation clusters are formed, as compared with the values of $\phi_{\mathrm{c}}$ in the swollen state and for the random close packing of hard spheres.
\end{abstract}

Polymer Journal (2016) 48, 1079-1086; doi:10.1038/pj.2016.79; published online 14 September 2016

\section{INTRODUCTION}

Microgels are colloidal-scale particles, which are composed of chemically cross-linked polymer networks that are swollen by solvents. ${ }^{1-6}$ Microgels are mechanically soft and they have fuzzy surfaces consisting of a swollen hairy layer in contrast to hard-sphere particles, which have well-defined surfaces. In the dilute regime, microgel suspensions behave as viscoelastic liquids in substantially the same way as hard-sphere suspensions. A unique feature of soft microgel suspensions is that they can be concentrated exceeding the volume fraction for random close packing, because the microgels can undergo deformation, interpenetration and/or deswelling in the highly dense states. These concentrated suspensions, which are called pastes, exhibit a solid-like behavior when the imposed stress and strain are sufficiently small. ${ }^{7-9}$ When the imposed stress or strain is beyond a critical value (yield stress or strain), the dense microgel suspensions exhibit non-Newtonian fluid behavior. Importantly, the rheological characteristics of microgel suspensions such as modulus, viscosity and yield stress can be tuned by varying the microgel architectures such as cross-link concentration, particle size and surface. These interesting rheological features of the microgel suspensions have been used for various industrial applications such as paints, cosmetics and drug delivery systems. ${ }^{10}$

In recent years, microgels that respond to external stimuli such as temperature, $\mathrm{pH}$, flow, electric field and osmotic pressure have attracted much interest in academic and industrial areas. Poly ( $N$-isopropylacrylamide) (PNIPAM) is known as a representative thermo-responsive polymer. ${ }^{11}$ Aqueous solutions of PNIPAM have a lower critical solution temperature (LCST) of around $33^{\circ} \mathrm{C}$. The microhydrogels composed of PNIPAM chains undergo a volume transition at around the LCST as observed for the corresponding macroscopic hydrogels. ${ }^{12,13}$ The microgels are highly swollen at temperatures $(T)$ below a characteristic temperature $\left(T^{\star}\right)$ due to the hydration of PNIPAM and they are considerably shrunken in the $T>T^{*}$ range due to dehydration. These thermo-responsive properties of the PNIPAM microgels result in potential applications in medical and cosmetic fields, because $T^{*}$ is easily accessible and near body temperature. An intriguing feature of PNIPAM microgel suspensions is that the viscoelasticity is thermally controllable without changing the mass concentration of particles, because the $T$ variations change the volume fraction of the microgel particles in the suspensions.

${ }^{1}$ Department of Macromolecular Science and Engineering, Kyoto Institute of Technology, Kyoto, Japan; ${ }^{2}$ Graduate School of Textile Science and Technology, Shinshu University, Ueda, Japan and ${ }^{3}$ Division of Smart Textile, Institute for Fiber Engineering, Interdisciplinary Cluster for Cutting Edge Research, Shinshu University, Ueda, Japan

Correspondence: Professor D Suzuki, Graduate School of Textile Science and Technology, Shinshu University, Ueda 386-8567, Japan.

E-mail: d_suzuki@shinshu-u.ac.jp

or Professor K Urayama, Department of Macromolecular Science and Engineering, Kyoto Institute of Technology, Matsugasaki, Sakyo-ku Kyoto 606-8585, Japan.

E-mail: urayama@kit.ac.jp

Received 7 June 2016; revised 19 July 2016; accepted 19 July 2016; published online 14 September 2016 
Moreover, a merit of the microgel suspensions is that they can respond quickly to $T$ variations due to the rapid volume change of the submicrometer gel particles.

The thermal effects on the rheological properties of PNIPAM microgel suspensions have been reported by several researchers. ${ }^{14-20}$ In the swollen state of $T<T^{*}$, the dispersions are stabilized by repulsive interparticle interactions that originate from steric hindrance of dangling chains. Richtering et al. ${ }^{15,16}$ analyzed the effects of the $T$ and particle concentration on the viscosity and modulus of suspensions in the $T<T^{\star}$ range using the effective volume fraction of microgels $\left(\phi_{\text {eff }}\right)$ as a single variable. Romeo et al. ${ }^{19}$ investigated the linear dynamic viscoelasticity, that is, the frequency $(\omega)$ dependence of the storage modulus $\left(G^{\prime}\right)$ and loss modulus $\left(G^{\prime \prime}\right)$, for concentrated suspensions of polydisperse PNIPAM microgels in a wide $T$ range across $T^{*}$. At a low $T$ below $T^{*}$, the dense suspensions show an elastic response of $G^{\prime}>G^{\prime \prime}$ as a glass with repulsive interaction. These dense suspensions can liquefy by heating as a result of a decrease in $\phi_{\text {eff. }}$. They found that the suspensions exhibit an elastic response again when heated above $T^{\star}$, despite a considerable reduction in $\phi_{\text {eff. }}$ The origin of the elastic behavior in the $T>T^{*}$ range was due to the formation of a solid gel by attractive interactions between the collapsed microgels. Zhou et al. ${ }^{21}$ studied the nonlinear viscoelasticity of the dense suspensions below and beyond $T^{\star}$ for microgels composed of a polystyrene core and a PNIPAM shell.

The aggregated colloidal suspensions formed by attractive forces have received considerable attention as attractive glasses or colloidal gels and the rheological properties of the aggregated colloidal dispersions have been previously investigated by a number of authors. ${ }^{22-30}$ It is known that various types of colloidal particles with attractive forces form aggregated dispersions. In general, the aggregated suspensions exhibit broad relaxation spectra and they require a long time for flowing if they flow. ${ }^{22}$ The earlier rheological studies ${ }^{19,21}$ on the aggregated suspensions of PNIPAM-based microgels beyond $T^{*}$ observed an elastic response but their observations only relied on oscillatory measurements for a certain range of angular frequencies $(\omega)$, typically $\omega>0.01 \mathrm{~s}^{-1}$. However, the accessible time scale of oscillatory experiments is typically not as long as several hundred seconds. Rheological experiments with longer accessible time scales such as creep tests under constant stress are required to elucidate whether the aggregated dispersions flow or not at long times; in other words, whether they undergo definite solidification or not. The long-time scale measurements will also contribute to the characterization of the flow properties if they flow. However, to the best of our knowledge, no corresponding long-timescale experiments of the aggregated suspensions of the PNIPAM microgel have been previously reported. Interparticle aggregation by attractive forces is expected to significantly influence the viscosity of the suspensions in not only the concentrated but also dilute regimes. The viscosity in the dilute regime has been studied mainly in the $T<T^{\star}$ region where the repulsive interparticle interactions are dominant. ${ }^{15,17}$ It still remains to be characterized how the viscosity of dilute suspensions depends on the particle concentration in the regime of $T>T^{\star}$ regime.

In the current study, we mainly focus on the linear viscoelasticity of PNIPAM microgel suspensions with a wide range of particle concentrations in the $T<T^{*}$ and $T>T^{*}$ regimes, that is, where the repulsive and attractive interparticle interactions are dominant, respectively. In particular, the long-time creep measurements reveal the terminal flow behavior of the aggregated microgel dispersions with a high viscosity and long characteristic time for flowing. The results in the current study will contribute to a deeper understanding of the rheological properties of microgel suspensions with repulsive and attractive interparticle interactions, and they will provide important information and knowledge about the molecular design of microgel suspensions with temperature-controllable viscoelasticity.

\section{MATERIALS AND METHODS}

\section{Materials}

NIPAM (purity 98\%), $N, N^{\prime}$-methylenebis (acrylamide) (97\%), SDS (95\%) and potassium peroxodisulfate $(95 \%)$ were purchased from Wako Pure Chemical Industries (Osaka, Japan) and used as received. The water used for the microgel synthesis and purification was distilled and then ion-exchanged (EYELA, SA-2100E1, Tokyo, Japan) before use.

\section{Synthesis of PNIPAM microgels}

Chemically cross-linked PNIPAM microgels were synthesized via aqueous free radical precipitation polymerization using the water-soluble anionic initiator potassium peroxodisulfate. ${ }^{31}$ The initial total monomer concentration was held constant at $150 \mathrm{~mm}$. A mixture of NIPAM $(8.0630 \mathrm{~g}, 95 \mathrm{~mol} \%)$, the cross-linker $N, N^{\prime}$-methylenebis (acrylamide) $(0.5783 \mathrm{~g}, 5 \mathrm{~mol} \%$ ), the anionic surfactant SDS $(0.2884 \mathrm{~g}, 2 \mathrm{~mm})$ and water $(295 \mathrm{ml})$ was poured into a 1 liter three-neck round-bottom flask equipped with a mechanical stirrer, condenser and nitrogen gas inlet. The monomer solution was heated to $70^{\circ} \mathrm{C}$ under a stream of nitrogen gas with constant stirring at 250 r.p.m. and then stabilized for $30 \mathrm{~min}$ to purge oxygen dissolved in the solution. The initiator potassium peroxodisulfate $(0.2706 \mathrm{~g}, 2 \mathrm{~mm})$ dissolved in $5 \mathrm{ml}$ of water was injected into the flask to initiate the free-radical polymerization. The reaction was allowed to proceed for $4 \mathrm{~h}$ and the resultant microgel suspensions were then allowed to cool to room temperature. To remove unreacted chemicals and surfactants, these microgels were purified twice via centrifugation/redispersion with water using a relative centrifugal force of $415,000 \mathrm{~g}$ and by means of dialysis for 7 days with daily changes of water. The dense suspensions were obtained by concentrating the suspensions using centrifugation $(415,000 \mathrm{~g})$.

\section{Measurements}

The hydrodynamic diameter $\left(D_{\mathrm{h}}\right)$ of the gel particle in the isolated state was evaluated as a function of temperature in the range between $25^{\circ} \mathrm{C}$ and $45^{\circ} \mathrm{C}$ using a dilute suspension of $c=2.53 \times 10^{-5} \mathrm{~g} \mathrm{ml}^{-1}$ using dynamic light scattering (Malvern Instruments; ZetasizerNanoS, Malvern, UK). The dynamic light scattering data correspond to an average of 15 independent measurements of the intensity auto correlation acquired over $30 \mathrm{~s}$ and the average of the results for three measurements. The sample was allowed to equilibrate for $10 \mathrm{~min}$ at a set temperature before measurements. The ionic strength was adjusted to $1 \mathrm{~mm}$ with $\mathrm{NaCl}$.

The intrinsic viscosities ([ $\eta])$ of the microgel particles at various temperatures were estimated from the viscosities of the sufficiently diluted suspensions using an Ubbelohde viscometer based on Huggins and Mead-Fuoss plots.

The rheological measurements were performed with a stress-controlled AR-G2 (TA Instruments, New Castle, DE, USA) rheometer using a cone-plate geometry $\left(40 \mathrm{~mm}\right.$ diameter and $1^{\circ}$ cone angle). The measurements were performed with a sufficiently small stress or stress amplitude that was well inside the linear regime unless specified otherwise. The fixture was covered with a lid filled with water, to prevent water evaporation during the measurements. All of the measurements were performed after equilibrating the specimens at constant temperature.

\section{RESULTS AND DISCUSSION}

\section{Characterization of isolated PNIPAM microgel particles}

Figure 1 shows the temperature $(T)$ dependence of $D_{\mathrm{h}}$ for the isolated PNIPAM microgel particles. The diameter $D_{\mathrm{h}}$ decreases with an increase in $T$ along with a sharp change in the range between $32^{\circ} \mathrm{C}$ and $36^{\circ} \mathrm{C}$. In the regime of $T>36^{\circ} \mathrm{C}, D_{\mathrm{h}}$ is insensitive to $T$. The diameter $D_{\mathrm{h}}$ at $45^{\circ} \mathrm{C}$ in the collapsed state is approximately half of that at $25^{\circ} \mathrm{C}$ in the swollen state. The volume phase-transition temperature $\left(T^{\star}\right)$ is estimated to be $33.5^{\circ} \mathrm{C}$, where the gradient of 
the $D_{\mathrm{h}}-T$ curve becomes maximum. As is shown in Figure $1,[\eta]$ varies with $T$ in a qualitatively the same way as $D_{\mathrm{h}}$. As is evident from the definition, $[\eta]$ of the microgel particles is proportional to the degree of swelling, that is, the ratio of the volume in the swollen state to the weight in the dry state. Figure $1 \mathrm{~b}$ shows the relationship between $[\eta]$ and $D_{\mathrm{h}}{ }^{3}$ over the entire $T$ range. The relationship is well
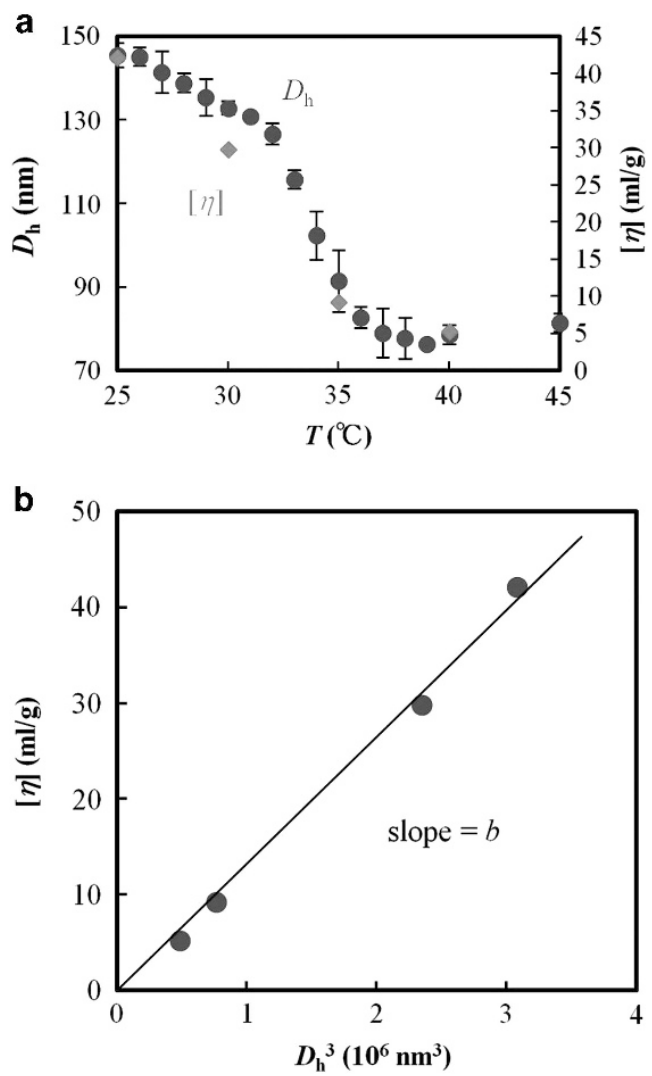

Figure 1 (a) $T$ dependence of $D_{\mathrm{h}}$ and $[\eta]$ for the PNIPAM microgel particles. (b) $[\eta]$ as a function of $D_{\mathrm{h}}^{3}$ at various $T$. The proportional constant $b$ is $1.32 \times 10^{16} \mathrm{~g}^{-1}$. A full color version of this figure is available at the Polymer Journal online. approximated by a linear correlation, that is, $[\eta]=b D_{\mathrm{h}}{ }^{3}$ with a proportional constant $b$.

As it is customary to do when dealing with microgel suspensions, the effective volume fraction of particles $\left(\phi_{\text {eff }}\right)$ that is defined by equation (1) is used here as a measure of the degree of packing independent of the concentration regime: ${ }^{32-37}$

$$
\phi_{\text {eff }}=\frac{c[\eta]}{2.5}
$$

where $c$ is the particle concentration. The linear relationship between $[\eta]=b D_{\mathrm{h}}{ }^{3}$ (Figure 1b) and equation (1) leads to the following equation:

$$
\phi_{\mathrm{eff}}=b \frac{c D_{\mathrm{h}}{ }^{3}}{2.5}
$$

Equation (2) and the $D_{\mathrm{h}}-T$ data in Figure 1a enable us to evaluate $\phi_{\text {eff }}$ for the suspensions at given values of $T$ and $c$. It is worth noting that $\phi_{\text {eff, }}$ which is estimated from the volume of the isolated particle, differs from the real volume fraction in the concentrated regime of $\phi_{\text {eff }}>\phi_{c}$, where the microgels can undergo deformation, deswelling and/or interpenetration and $\phi_{\text {eff }}$ is used here as an estimation to analyze the data.

Temperature dependence of the linear dynamic viscoelasticity of dense suspensions

Figure 2 shows the storage modulus $\left(G^{\prime}\right)$ and loss modulus $\left(G^{\prime \prime}\right)$ for a highly concentrated suspension with $c=0.0822 \mathrm{~g} \mathrm{ml}^{-1}$ at various $T$ as a function of the angular frequency $(\omega)$, which ranged from 0.1 to $100 \mathrm{~s}^{-1}$. To avoid overlapping, the data at each $T$ are horizontally shifted by a factor of $A$. This suspension at low $T$ is in a highly dense state, which is evident from the high value of $\phi_{\text {eff }}\left(\phi_{\text {eff }}=1.34\right.$ at $\left.25^{\circ} \mathrm{C}\right)$ that exceeds unity. Varying $T$ for a suspension (with fixed $c$ ) corresponds to varying $\phi_{\text {eff }}$ with keeping the total number of microgels unchanged. The suspensions at 25 and $28^{\circ} \mathrm{C}$ exhibit elastic behavior: $G^{\prime}$ is nearly independent of $\omega$ and approximately one order of magnitude higher than $G^{\prime \prime}$. At $32^{\circ} \mathrm{C}$, the behavior becomes considerably viscoelastic: both $G^{\prime}$ and $G^{\prime \prime}$ are frequency dependent and they are almost identical in the $\omega<10^{1} \mathrm{~s}^{-1}$ range. In the swollen state at $T<T^{*}$, both moduli decrease with an increase in $T$. At $35^{\circ} \mathrm{C}$ above $T^{*}$, the suspension shows elastic behavior again, despite the considerably

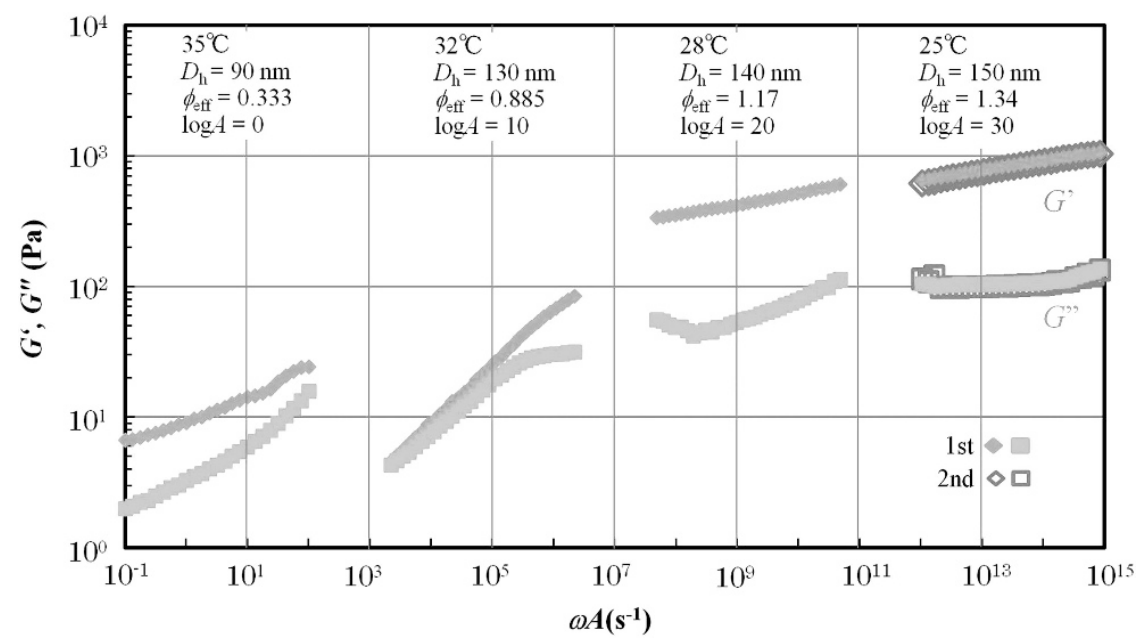

Figure $2 \omega$ dependence of $G^{\prime}$ and $G^{\prime \prime}$ for the suspensions of $c=0.0822 \mathrm{~g} \mathrm{ml}^{-1}$ at various temperatures. The data at each $T$ are horizontally shifted by a factor of $A$ to avoid overlapping. The open symbols denote the data which were re-measured at $25^{\circ} \mathrm{C}$ after the measurements at $35^{\circ} \mathrm{C}$. $\mathrm{A}$ full color version of this figure is available at the Polymer Journal online. 
low $\phi_{\text {eff }}$ value $\left(\phi_{\text {eff }}=0.333\right): G^{\prime}$ is larger than $G^{\prime \prime}$ in the whole $\omega$ range investigated here and $G^{\prime}$ is higher than that at $32^{\circ} \mathrm{C}$ in the low $\omega$ range of $\omega<1 \mathrm{~s}^{-1}$. The observed increase in elastic character on heating across $T^{*}$ is qualitatively similar to that observed in previous oscillatory tests. ${ }^{19,21}$ The details of the elastic behavior in the collapsed state will be discussed together with the data from the creep and recovery tests in a later section. Figure 2 also involves the data (denoted by open symbols) re-measured at $25^{\circ} \mathrm{C}$ after the measurement at $35^{\circ} \mathrm{C}$. The agreement of the data before and after heating above $T^{*}$ indicates that the structural changes in the suspensions between the swollen and collapsed states are thermally reversible.

\section{Equilibrium shear modulus of dense suspensions below $T^{*}$}

The dense suspensions with sufficiently high $\phi_{\text {eff }}$ exhibit a quasiplateau shear modulus $\left(G_{0}\right)$ in the low $\omega$ range and a change in $T$ causes a variation in $G_{0}$, which is shown in Figure 2. We focus on the effects of $T$ and $c$ on $G_{0}$ in the swollen state at $T<T^{*}$. Figure 3 shows a double-logarithmic plot of $G_{0}$ as a function of $\phi_{\text {eff }}$ using the data obtained for the dense suspensions with three different particle concentrations at various $T$ below $T^{*}$ and the $\phi_{\text {eff }}$ values for the specimens examined were larger than 1.1. The modulus $G_{0}$ increases with an increase in $\phi_{\text {eff }}$ and all of the data at various values of $T$ and $c$ fall on a single straight line with a slope of 4.0 , that is, $G_{0} \sim \phi_{\text {eff }}{ }^{m}$ with $m=4.0$. Senff et al. ${ }^{16}$ observed a similar relationship and they reported that the power-law exponent $m(3.19 \leq m \leq 5.82)$ increased as the cross-link density increased. This scaling relationship between $G_{0}$ and $\phi_{\text {eff }}$ indicates that $G_{0}$ is solely governed by the degree of packing and independent of $D_{\mathrm{h}}$, the number of particles and T. However, this relationship is not simply validated, because a thermally induced change in $D_{\mathrm{h}}$ also varies the polymer density inside the microgels, resulting in a change in the stiffness of the particles. The variation in $D_{\mathrm{h}}$ in the $T$ range of interest $\left(25^{\circ} \mathrm{C}<T<30^{\circ} \mathrm{C}\right)$ is not larger than $15 \%$ (Figure 1a) and thus the corresponding change in the particle stiffness will be negligibly small.

Long-time creep behavior of aggregated suspensions above $T^{\star}$ As shown in Figure 2, the suspension at $35^{\circ} \mathrm{C}\left(>T^{*}\right)$ exhibits much more elastic behavior than that at $32^{\circ} \mathrm{C}\left(<T^{*}\right)$ despite a considerable reduction in $\phi_{\text {eff. }}$. In order to exclusively discuss the effects of the type of interparticle interactions (that is, repulsive or attractive) on the

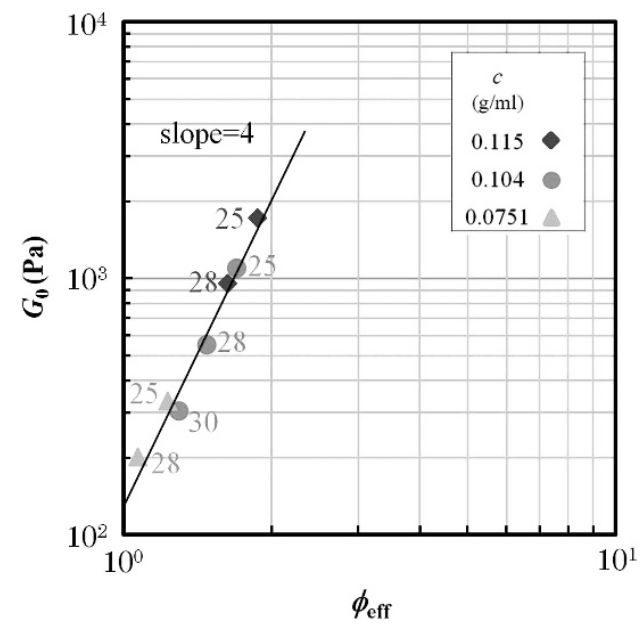

Figure $3 \phi_{\text {eff }}$ dependence of $G_{0}$ for the dense suspensions with various $C$ and $T$ in the swollen state of $T<T^{*}$. The numerals indicate the temperature. A full color version of this figure is available at the Polymer Journal online. viscoelasticity, the two suspensions with different $c$ values but the same $\phi_{\text {eff }}\left(\phi_{\text {eff }} \approx 0.46\right)$ at 25 and $35^{\circ} \mathrm{C}$ are employed for the measurements: $c=0.0283 \mathrm{~g} \mathrm{ml}^{-1}$ for $25^{\circ} \mathrm{C}$ and $c=0.115 \mathrm{~g} \mathrm{ml}^{-1}$ for $35^{\circ} \mathrm{C}$. Figure 4 shows the $\omega$ dependence of the linear dynamic moduli of the suspensions for $\phi_{\mathrm{eff}} \approx 0.46$ at $25^{\circ} \mathrm{C}$ and $35^{\circ} \mathrm{C}$. The suspension at $25^{\circ} \mathrm{C}$ exhibits typical Newtonian flow behavior, that is, the relationship of $G^{\prime \prime}>G^{\prime}, G^{\prime} \sim \omega^{2}$ and $G^{\prime} \sim \omega^{1}$. In contrast, the suspension at $35^{\circ} \mathrm{C}$ shows elastic behavior: $G^{\prime}$ is nearly independent of $\omega$ and the $G^{\prime}>G^{\prime \prime}$ relationship is observed for the $\omega$ range examined here.

The creep and recovery measurements, which have timescales that are much longer than the oscillatory measurements, are conducted in order to confirm whether the suspension with $\phi_{\text {eff }} \approx 0.46$ at $35^{\circ} \mathrm{C}$ flows at long times or not. Figure 5 displays the creep and recovery curves for the same samples shown in Figure 4. The creep compliance $\left(J: J=\gamma / \sigma_{0}\right.$, where $\gamma$ and $\sigma_{0}$ are the strain and imposed stress, respectively) was measured as a function of time $(t)$ under a protocol where a constant stress was imposed in the region of $0<t<t^{*}$ and, thereafter, the imposed stress was removed. The $\sigma_{0}$ value is the same as the stress amplitude used in the dynamic measurements in Figure 4 and this value is sufficiently small to be in the linear regime. In the swollen state at $25^{\circ} \mathrm{C}, J$ linearly increases with $t$ in the creep process and exhibits no recovery after removal of the stress (Figure $5 \mathrm{a}$ ). This result also confirms that a suspension with $\phi_{\text {eff }} \approx 0.46$ at $25^{\circ} \mathrm{C}$ behaves as a Newtonian fluid. The zero shear viscosity $\left(\eta_{0}\right)$ was evaluated from the inverse of the slope of the line to be $1.3 \times 10^{-2}$ Pas, which is nearly identical to the value obtained from the $G^{\prime \prime}$ data in Figure 4, that is, $\eta_{0}=1.5 \times 10^{-2}$ Pas $\left(\eta_{0}=G^{\prime \prime} / \omega\right)$.

The features of the creep and recovery data in the collapsed state at $35^{\circ} \mathrm{C}$ obviously indicate that the suspension can be classified as a viscoelastic 'liquid' with high viscosity that exhibits viscous flow at sufficiently long times (Figure 5b). The suspension exhibited instantaneous elastic response when the stress is imposed or removed. However, a linear increase in $J$ with $t$, that is, steady-state flow, is observed in the sufficiently long time region during the creep process. After the creep process reached a steady state, the imposed stress was removed at $t^{*}\left(t^{*}=1.8 \times 10^{3} \mathrm{~s}\right)$. A significant degree of residual strain (ca. $50 \%$ ) is observed in the steady state of the creep recovery, which is a clear indication of flow. The data for the second run of the creep and recovery tests, which was conducted immediately after the first run, were in agreement with those of the first run, as shown in Figure 5. This result confirms the reproducibility of the linear viscoelastic response in the collapsed state.

The zero shear viscosity $\left(\eta_{0}\right)$ at $35^{\circ} \mathrm{C}$ is evaluated from the creep data in the steady state to be $1.1 \times 10^{5} \mathrm{Pas}$, which is approximately seven orders of magnitude higher than that at $25^{\circ} \mathrm{C}$. The steady-state compliance $\left(J_{\mathrm{e}}\right)$ is estimated from the recovery data in the steady state by subtracting the instantaneous component from the recoverable compliance $\left(J_{R}\right)$. From a relation of $\tau_{\mathrm{L}}=\eta_{0} J_{\mathrm{e}}{ }^{38}$ the longest characteristic time for flow $\left(\tau_{\mathrm{L}}\right)$ at $35^{\circ} \mathrm{C}$ is obtained as $\tau_{\mathrm{L}}=1.2 \times 10^{3} \mathrm{~s}$, which is approximately five orders of magnitude longer than that of $25^{\circ} \mathrm{C}$. This long characteristic time for flow at $35^{\circ} \mathrm{C}$ explains why the terminal relaxation is not detected in the dynamic measurement (Figure 4): the corresponding characteristic frequency, which is given by $\tau_{\mathrm{L}}{ }^{-1}$ $\left(8.3 \times 10^{-4} \mathrm{~s}^{-1}\right)$, is much lower than the accessible lowest $\omega$ (typically $10^{-2} \mathrm{~s}^{-1}$ ) in the oscillatory measurements. The inverse of $J_{\mathrm{R}}$ $\left(J_{\mathrm{R}}=2.7 \times 10^{-2} \mathrm{~Pa}^{-1}\right)$ is nearly comparable to the quasi-plateau value of $G^{\prime}(c a .100 \mathrm{~Pa})$ in Figure 4. This result indicates that the elastic contribution that is observed in the creep recovery test could almost be explained by the magnitude of the quasi-plateau modulus of $G^{\prime}$. Notably, the sufficiently dense suspensions in the swollen state of $T<T^{*}$, which show the elastic response in oscillatory tests (Figure 2), 


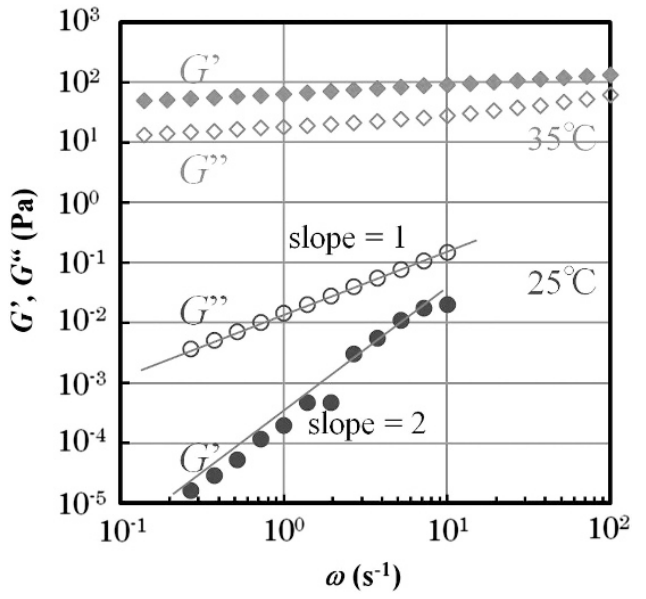

Figure 4 Linear dynamic viscoelasticity for the suspensions of $\phi_{\text {eff }}=0.461$ at $25^{\circ} \mathrm{C}\left(c=0.0283 \mathrm{~g} \mathrm{ml}^{-1}\right)$ and $\phi_{\text {eff }}=0.465$ at $35^{\circ} \mathrm{C}\left(c=0.115 \mathrm{~g} \mathrm{ml}^{-1}\right)$. A full color version of this figure is available at the Polymer Journal online.
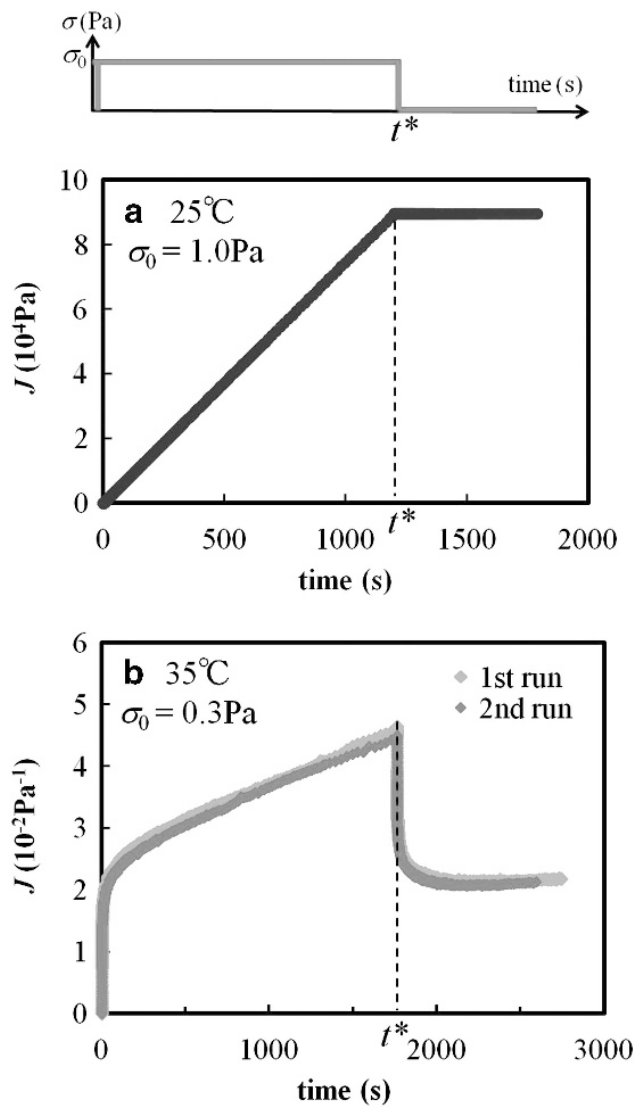

Figure 5 Time dependence of $J$ in the creep and creep recovery tests for the suspensions of $\phi_{\text {eff }} \approx 0.46$ at (a) $25^{\circ} \mathrm{C}$ and (b) $35^{\circ} \mathrm{C}$. The specimens are the same as in Figure 4. $t^{*}$ denotes the time at the removal of imposed stress and $t^{*}$ is $1.2 \times 10^{3} \mathrm{~s}$ at $25^{\circ} \mathrm{C}$ and $1.8 \times 10^{3} \mathrm{~s}$ at $35^{\circ} \mathrm{C}$. The imposed stress $\left(\sigma_{0}\right)$ is identical with the stress amplitude used for the linear dynamic measurements (Figure 4). The second run was conducted immediately after the first run. A full color version of this figure is available at the Polymer Journal online.

are classified into a viscoelastic solid with yield stress, because they exhibit perfect strain recovery in the creep recovery tests with a stress that is less than the yield stress, which was demonstrated in our previous study (Figure $3 \mathrm{~b}$ in Urayama et al. ${ }^{9}$ ).
In the collapsed state $\left(T>T^{*}\right)$, the interparticle interactions become attractive due to the hydrophobic character of the PNIPAM chains. The attractive interactions between the particles is expected to form a network-like aggregation. For aqueous solutions of the linear PNIPAM chains, a considerable increase in the viscosity was observed at around the LCST, which resulted from the formation of a physical network due to intermolecular hydrophobic associations. ${ }^{39,40}$ Some studies ${ }^{19,21}$ have reported an elastic response of $G^{\prime}>G^{\prime \prime}$ for PNIPAM-based microgel suspensions above $T^{*}$ in oscillatory measurements at frequencies of $\omega>0.01 \mathrm{~s}^{-1}$. Their $G^{\prime}$ data exhibit a finite positive dependence on $\omega$ without a definite plateau, which is qualitatively similar to the data in Figures 2 and 4. Based on the current results from the creep tests (Figure 5), we cannot exclude the possibility that the aggregated suspensions with $\phi_{\text {eff }}$ values ranging from 0.2 to 0.6 may flow at long times in their studies. The suspensions with sufficiently high $\phi_{\text {eff }}$ in the collapsed state are expected to form a more developed aggregation that does not flow even at long times as in the case of different types of aggregated suspensions. ${ }^{41}$ This definite solidification was not confirmed in the current study, because the suspension with $c=0.115 \mathrm{~g} \mathrm{ml}^{-1}$ was the most concentrated one, that is, $\phi_{\text {eff }} \approx 0.46$ was the highest $\phi_{\text {eff }}$ accessible at $35^{\circ} \mathrm{C}$ ). Further concentration by strong centrifugal force caused an irreversible change in the specimens: The suspension obtained by diluting such a concentrated specimen was different in viscosity from that with the same particle concentration that was not subjected to centrifugal force.

Strength of particle aggregations above $T^{\star}$

The effects of the stress amplitude $\left(\sigma_{\mathrm{a}}\right)$ on the dynamic viscoelasticity for the suspension with $\phi_{\text {eff }} \approx 0.46$ at $35^{\circ} \mathrm{C}$ are investigated, in order to characterize the mechanical strength of the particle aggregations (Figure 6). In this experiment, the $\sigma_{\mathrm{a}}$ scan started from the lowest value $(0.01 \mathrm{~Pa})$. In the sufficiently small $\sigma_{\mathrm{a}}$ regime, a linear response are observed: both moduli are independent of $\sigma_{\mathrm{a}}$ and the strain amplitude $\left(\gamma_{\mathrm{a}}\right)$ linearly varied with $\sigma_{\mathrm{a}}$. The results confirmed in the figure that the stress employed in the oscillatory and creep measurements $\left(\sigma_{\mathrm{a}}=\sigma_{0}=0.3 \mathrm{~Pa}\right)$ shown in Figures 4 and 5 is within the linear regime. In this linear regime, $G^{\prime}$ is approximately four times larger than $G^{\prime \prime}$ and the suspension exhibits an elastic response of $G^{\prime}>G^{\prime \prime}$ at $\omega=0.1 \mathrm{~s}^{-1}$. When $\sigma_{\mathrm{a}}$ exceeds a critical stress $\left(\sigma_{\mathrm{c}}: \sigma_{\mathrm{c}} \approx 2 \mathrm{~Pa}\right)$, a steep drop of $G^{\prime}$ occurs and $G^{\prime}$ becomes less than $G^{\prime \prime}$, whereas the $\sigma_{\text {a }}$ dependence of $\gamma_{\mathrm{a}}$ becomes stronger. These results indicate that the network-like aggregation of particles is so fragile that it can be broken by a very small stress on the order of several Pascals. In fact, the direct appreciation of the high $\eta_{0}$ of the aggregated suspensions is difficult, because even very small degrees of shake and tilting can destroy the network-like aggregation, resulting in a marked reduction in viscosity due to the extremely small $\sigma_{\mathrm{c}}$ value and the strong shear-thinning effect at stresses beyond $\sigma_{\mathrm{c}}$.

The perfect recovery of the aggregated structure after the destruction by the imposed stress is also shown in Figure 6. The second run of the $\sigma_{\mathrm{a}}$ scan was performed $5 \mathrm{~min}$ after the measurement at the highest $\sigma_{\mathrm{a}}\left(3 \mathrm{~Pa}>\sigma_{\mathrm{c}}\right)$. The agreement of the data from the first and second runs confirms the reformation of the original aggregated structure after the destruction.

\section{Effect of temperature on viscoelasticity above $T^{\star}$}

The dynamic viscoelasticities of the two suspensions with nearly the same $\phi_{\text {eff }}\left(\phi_{\text {eff }} \approx 0.3\right)$ at $35^{\circ} \mathrm{C}$ and $40^{\circ} \mathrm{C}$ is compared, in order to investigate exclusively the effect of temperature on the particle aggregations: the concentrations of the two suspensions are $c=0.0822$ and $0.115 \mathrm{~g} \mathrm{ml}^{-1}$, which correspond to $\phi_{\text {eff }}=0.333$ and 


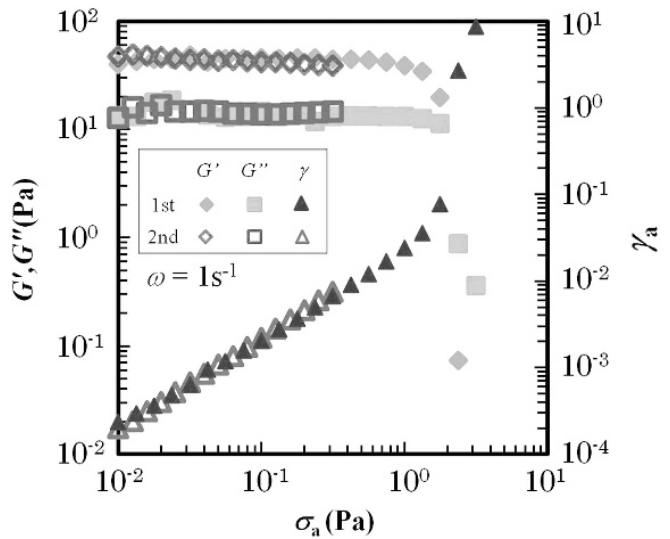

Figure $6 \sigma_{\mathrm{a}}$ dependence of $G^{\prime}$ and $G^{\prime \prime}$ for a suspension of $\phi_{\text {eff }}=0.465$ at $35^{\circ} \mathrm{C}\left(c=0.115 \mathrm{~g} \mathrm{ml}^{-1}\right)$. The second run was conducted $5 \mathrm{~min}$ after the measurements at the highest $\sigma_{\mathrm{a}}(3 \mathrm{~Pa})$. A full color version of this figure is available at the Polymer Journal online.
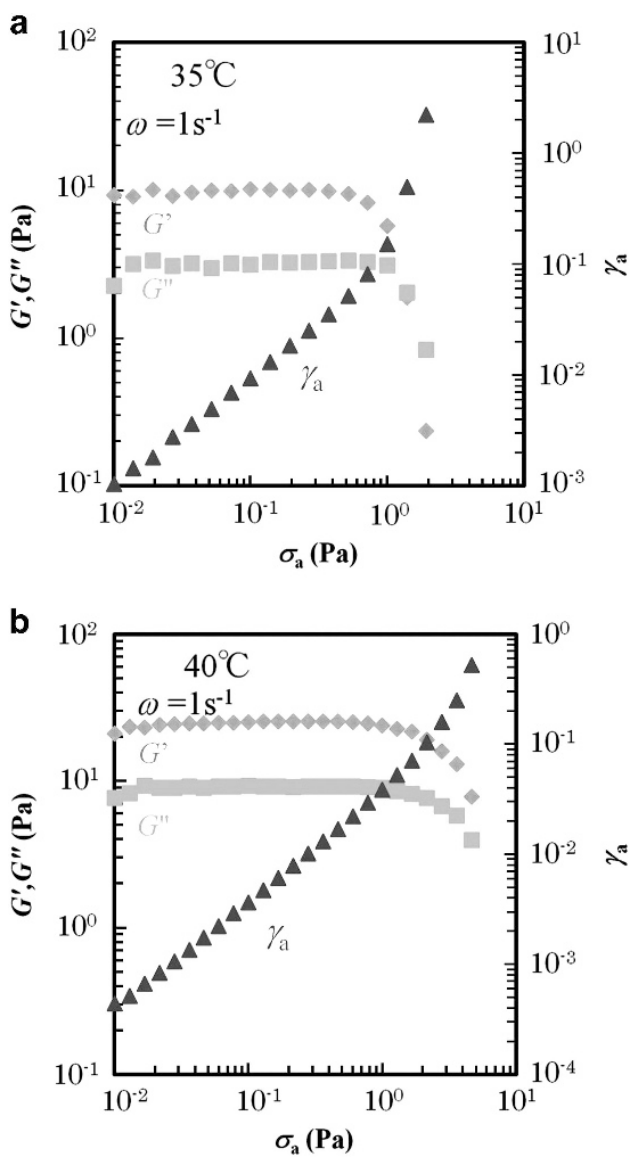

Figure $7 \sigma_{\mathrm{a}}$ dependence of $G^{\prime}$ and $G^{\prime \prime}$ for the suspensions at $35^{\circ} \mathrm{C}$ and $40{ }^{\circ} \mathrm{C}$ with almost the same $\phi_{\text {eff }}(\approx 0.3)$ : (a) $\phi_{\text {eff }}=0.333$ at $35^{\circ} \mathrm{C}$ $\left(c=0.0822 \mathrm{~g} \mathrm{ml}^{-1}\right)$ and (b) $\phi_{\text {eff }}=0.296$ at $40^{\circ} \mathrm{C}\left(c=0.115 \mathrm{~g} \mathrm{ml}^{-1}\right)$. A full color version of this figure is available at the Polymer Journal online.

0.296 at $35^{\circ} \mathrm{C}$ and $40{ }^{\circ} \mathrm{C}$, respectively. Figures 7 and 8 illustrate the dependence of the dynamic moduli on $\sigma_{\mathrm{a}}$ and $\omega$ at each temperature, respectively. Although the $\phi_{\text {eff }}$ values are nearly the same $\left(\phi_{\text {eff }} \approx 0.3\right)$, $G^{\prime}$ in the linear regime at $40^{\circ} \mathrm{C}$ is approximately four times higher than that at $35^{\circ} \mathrm{C}$. This result indicates that the elastic modulus in the collapsed state depends on not only $\phi_{\text {eff }}$ but also $T$, which is in contrast to the modulus in the swollen state that is solely governed by $\phi_{\text {eff }}$ (Figure 3). The results in Figure 7 indicate that $\sigma_{\mathrm{c}}$ at $40{ }^{\circ} \mathrm{C}$ is approximately two times higher than that at $35^{\circ} \mathrm{C}$. The particle aggregations in the collapsed state become more elastic and robust as $T$ increases. ${ }^{19,21}$ The increase in the modulus and robustness are attributed to the growth of attractive interactions between the microgels, which originates from an increase in the hydrophobicity of the PNIPAM chains.

\section{Zero shear viscosity at various temperatures}

As mentioned in a former section, the suspension with $\phi_{\text {eff }} \approx 0.46$ in the collapsed state exhibit a markedly higher viscosity due to the particle aggregations than the suspension with the same $\phi_{\text {eff }}$ in the swollen state. This section focuses on the $T$ effects on the $\phi_{\text {eff }}$ dependence of the zero shear viscosity $\left(\eta_{0}\right)$ in each state.

Figure 9 shows the $\phi_{\text {eff }}$ dependence of the relative zero shear viscosity $\left(\eta_{0, \mathrm{r}}=\eta_{0} / \eta_{\mathrm{w}}\right.$ where $\eta_{\mathrm{w}}$ is viscosity of water $)$ at various $T$. Creep tests were employed to measure the high viscosities of the suspensions with long-terminal relaxation times. The viscosity obtained from the creep tests is an average value of several measurements and the error bars denote the s.d. The data at $25^{\circ} \mathrm{C}$ and $30^{\circ} \mathrm{C}$ below $T^{*}$ fall on a single curve. This result indicates that $\eta_{0}$ in the swollen state is only governed by $\phi_{\text {eff }}$ irrespective of $T$ and $D_{\mathrm{h}}$, which was also observed in Senff and Richtering ${ }^{15}$, and Stieger et al. ${ }^{17}$ The dashed line in Figure 9 shows the familiar Dougherty-Krieger equation, which fairly well describes the $\phi$ dependence of $\eta_{0}$ for polydisperse hard sphere suspensions: ${ }^{42-44} \eta_{0, \mathrm{r}}=\left(1-\phi / \phi_{\mathrm{c}}\right)^{-2}$ where $\phi_{\mathrm{c}}$ is the threshold value for random close packing $\left(\phi_{\mathrm{c}}=0.64\right)$. The threshold $\phi_{\mathrm{c}}$ where $\eta_{0}$ diverges in the swollen state, which is estimated to be 0.93 by empirical fitting using the equation of $\eta_{0, \mathrm{r}}=\left(1-\phi / \phi_{\mathrm{c}}\right)^{-a}$ (depicted by the solid line), is appreciably higher than $\phi_{\mathrm{c}}$ for hard sphere suspensions. A higher shift of $\phi_{\mathrm{c}}$ was also observed for suspensions of some microgels with low cross-link densities. ${ }^{35,37}$ The microgels with low cross-link densities have low polymer densities due to high degrees of swelling. This allows the microgels to interpenetrate into each other at high $\phi_{\text {eff }}$ that results in a higher shift in $\phi_{c}$.

The viscosities $\eta_{0}$ in the collapsed state $\left(35\right.$ and $\left.40^{\circ} \mathrm{C}\right)$ are significantly higher than those in the swollen state $\left(25^{\circ} \mathrm{C}\right.$ and $\left.30^{\circ} \mathrm{C}\right)$ when compared at the same $\phi_{\text {eff. }}$ In addition, the two suspensions with $\phi_{\text {eff }}=0.297$ at $40{ }^{\circ} \mathrm{C}$ and $\phi_{\text {eff }}=0.398$ at $35^{\circ} \mathrm{C}$ exhibit nearly the same $\eta_{0}$ (76.1 and 69.8 Pas, respectively), although $\phi_{\text {eff }}$ at $40^{\circ} \mathrm{C}$ is appreciably smaller than that at $35^{\circ} \mathrm{C}$. These results indicate that $\eta_{0}$ in the collapsed state depends on both $\phi_{\text {eff }}$ and $T$, and that $\eta_{0}$ becomes higher as $T$ increases. The $\phi_{\mathrm{c}}$ value at $35^{\circ} \mathrm{C}$ is estimated to be 0.51 by the empirical fitting described before (depicted by the solid line). The $\phi_{\mathrm{c}}$ value at $35^{\circ} \mathrm{C}$ is considerably smaller than those in the swollen state $\left(\phi_{c}=0.93\right)$ and for random close packing of hard spheres $\left(\phi_{c}=0.64\right)$. This behavior is explained by the following features in the collapsed state: The microgel particles are not allowed to interpenetrate into each other due to the high polymer density in the surface layer. In addition, the attractive interactions result in a strong correlation for the connection of different particles, which enables the formation of infinite percolation clusters at lower particle concentrations. These features are consistent with previous reports that observed the formation of clusters at low $\phi_{\text {eff }}$ in PNIPAM microgel suspensions. ${ }^{45}$ A previous study reported a monotonic decrease in viscosity with as $T$ increased across $T^{\star}$ without anomaly for dilute and moderately concentrated PNIPAM suspensions. ${ }^{39}$ Although the reason for no appreciable effect of the phase transition on the viscosity is unclear, the viscosity data in their study may be subjected to the strong 


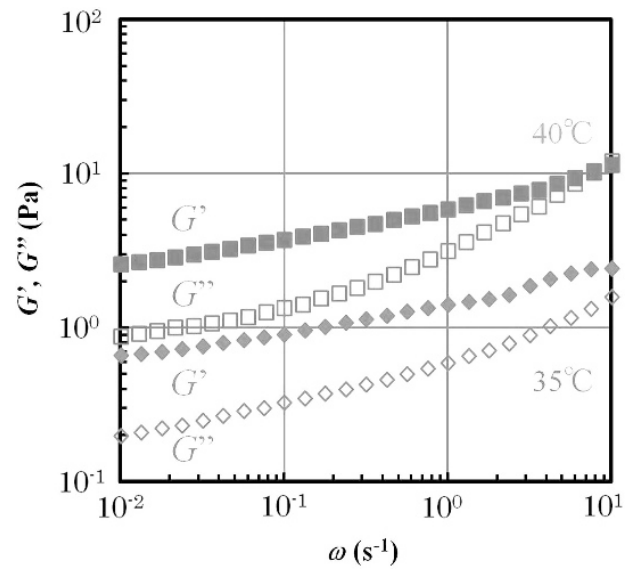

Figure 8 Linear dynamic viscoelasticity for the suspensions at $35^{\circ} \mathrm{C}$ and $40^{\circ} \mathrm{C}$ with almost the same $\phi_{\text {eff }}(\approx 0.3): \quad \phi_{\text {eff }}=0.333$ at $35^{\circ} \mathrm{C}$ $\left(c=0.0822 \mathrm{~g} \mathrm{ml}^{-1}\right)$ and $\phi_{\mathrm{eff}}=0.296$ at $40^{\circ} \mathrm{C}\left(c=0.115 \mathrm{~g} \mathrm{ml}^{-1}\right)$. A full color version of this figure is available at the Polymer Journal online.

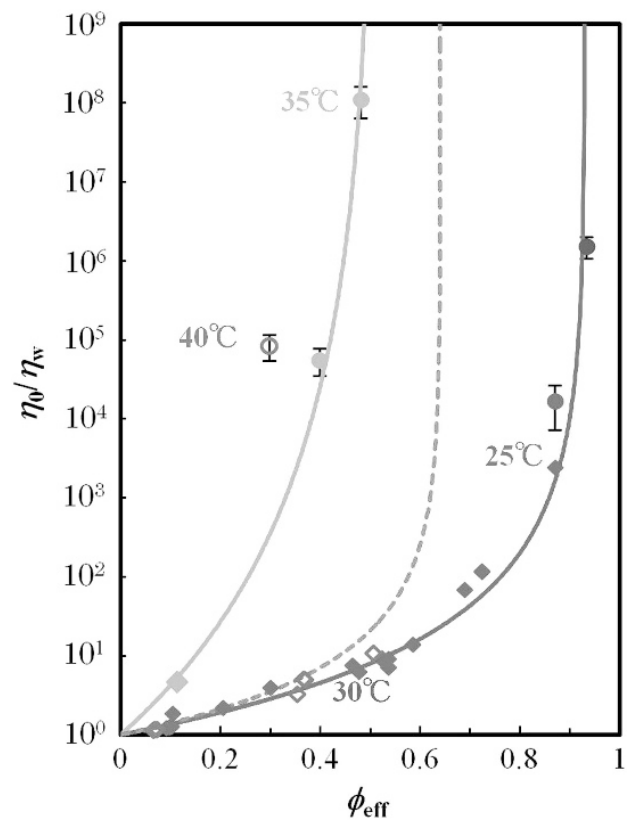

Figure $9 \phi_{\text {eff }}$ dependence of $\eta_{0} / \eta_{\mathrm{w}}$ for the suspensions at various $T$. The solid lines display the empirically fitted curves of $\eta_{0} / \eta_{\mathrm{w}}=\left(1-\phi / \phi_{\mathrm{c}}\right)^{-a}$ with $\phi_{\mathrm{C}}=0.93$ and $a=2.7$ at the temperatures of $T<T^{*}$, and with $\phi_{\mathrm{C}}=0.51$ and $a=6.6$ at $35^{\circ} \mathrm{C}\left(T>T^{*}\right)$. The dashed line depicts the Dougherty-Krieger relation for polydisperse hard spheres with $\phi_{\mathrm{C}}=0.64$ and $a=2.43,44$ The diamond and circular symbols denote the data obtained by oscillatory and creep experiments, respectively. A full color version of this figure is available at the Polymer Journal online.

shear-thinning effect in a non-Newtonian region: The time scale $(1 \mathrm{~s})$ corresponding to the minimum shear rate $\left(1 \mathrm{~s}^{-1}\right)$ in their study is much shorter than the terminal relaxation time for the aggregated suspensions estimated here $\left(\tau_{\mathrm{L}} \approx 10^{3} \mathrm{~s}\right.$ for $\phi_{\text {eff }} \approx 0.46$ at $\left.35^{\circ} \mathrm{C}\right)$.

The features for the dependence of $\eta_{0}$ on $\phi_{\text {eff }}$ and $T$ observed in each state are qualitatively similar to those for the elastic modulus. In the swollen state, where the interparticle interaction is governed by repulsive forces, the viscoelastic properties of the suspensions solely depend on $\phi_{\text {eff }}$ independently of whether they are in a dilute or highly concentrated state. In the collapsed state, where the attractive interaction is dominant, the viscoelasticity of the suspensions is influenced by both $\phi_{\text {eff }}$ and $T$. The network-like aggregations of the collapsed microgels formed by attractive interactions enhance the viscosity and modulus. An attempt was made to observe the network-like aggregations but the optical microscope observation was precluded by the high turbidity of the suspensions.

No macroscopic precipitation is observed here in the collapsed state with attractive interactions. The stabilization of the suspensions in the collapsed state is due to the presence of electrostatic repulsion. The neutral PNIPAM microgel suspensions become colloidally unstable close to the LCST. ${ }^{15}$ In our microgels in the collapsed state, a significant degree of surface density with negative charge is confirmed by a finite electrophoretic mobility in the salt-free suspensions, which is shown in Supplementary Figure 1. This charge originates from the residues of potassium peroxodisulfate used as an initiator, which is attached to the end of the PNIPAM chains. ${ }^{31}$ An iridescent color, which indicates the formation of colloidal crystals, is not observed in the suspensions at $T>T^{*}$. The important role of the surface charge in the stabilization is further confirmed by the observation that the macroscopic precipitation was caused by addition of $\mathrm{NaCl}$ salt (corresponding to $2 \mathrm{~mm}$ ) in the suspension with $c=6.0 \times 10^{-3} \mathrm{~g} \mathrm{ml}^{-1}$ at $35^{\circ} \mathrm{C}$. Some studies also employed PNIPAM microgels with charged groups for stabilization of the colloidal suspensions in the collapsed state. ${ }^{14,19}$ However, the mechanism of the stabilization by charged groups in the collapsed state remains unclear. The weak particle aggregation observed in this study is expected to result from an appropriate degree of surface charge density that weakens the attractive interaction to prevent macroscopic precipitation. The surface charge density in the current shrunken gels is modest, because it only stems from the initiator residues. Our PNIPAM microgels exhibit a continuous volume transition (Figure 1), whereas the discontinuity in the transition becomes obvious in the macroscopic PNIPAM gels when the electrolyte units, such as acrylic acid monomers, are explicitly introduced into the network strands by copolymerization. ${ }^{13}$ The magnitude of the negative charge in the current microgels is too small to cause an appreciable discontinuous volume transition. Hirose et al. ${ }^{13}$ also observed a continuous transition for PNIPAM microgels, where ammonium persulfate, which is similar to potassium peroxodisulfate in the current study, was used as an initiator. It is interesting to explore how further increases in the surface charge density influences the aggregation or dispersion state of the collapsed microgels, which will be investigated in our future study.

In the swollen state, the repulsive interaction that is driven by steric hindrance of the dangling chains mainly contributes to stabilization of the suspensions. As demonstrated in the electrophoretic mobility data (see Supplementary Figure 1), the surface charge density in the swollen state is considerably suppressed relative to that in the collapsed state as a consequence of the dilution effect by high swelling. No macroscopic precipitation occurred when the same amount of salt as that described above was added to the corresponding suspension in the swollen state.

\section{SUMMARY}

The rheological behavior of the suspensions of PNIPAM microgels with negative surface charges has been investigated in a wide range of $T$ across the volume phase transition temperature $\left(T^{\star}\right)$ using oscillatory tests in combination with long-time creep tests.

In the swollen state $\left(T<T^{*}\right)$ with repulsive interparticle interactions, the quasi-plateau shear modulus $\left(G_{0}\right)$ for highly dense suspensions as well as the zero shear viscosity for dilute suspensions are solely governed by $\phi_{\text {eff }}$ independent of temperature $(T)$, particle 
diameter and particle concentration, which is in agreement with the previously reported observations. ${ }^{15,16}$

When the dense suspensions, which exhibit elastic behavior with a quasi-plateau modulus in the $T<T^{*}$ regime, are heated above $T^{\star}$, they become viscoelastic liquids with high viscosities and long-terminal relaxation times of $>10^{3} \mathrm{~s}$ due to the formation of network-like aggregation by attractive interparticle interaction. These samples apparently exhibit elastic behavior with $G^{\prime}>G^{\prime \prime}$ in the oscillatory tests with relatively short experimental time scales, but their flow behavior is clearly observed in the long-time creep and recovery tests: the aggregated suspension with $\phi_{\text {eff }} \approx 0.46$ at $35^{\circ} \mathrm{C}\left(>T^{\star}\right)$ exhibits a zero shear viscosity higher than the suspension with the same $\phi_{\text {eff }}$ at $25^{\circ} \mathrm{C}$ $\left(<T^{\star}\right)$ by seven orders of magnitude. The modulus of the aggregated suspensions increases with an increase in temperature, whereas the particle aggregations are so fragile that they can be broken by a very small stress on the order of several Pascals. The particle aggregations show perfect recovery after destruction by the imposed stress.

In the collapsed state $\left(T>T^{*}\right)$ with attractive interparticle interactions, the modulus and viscosity depend on both $\phi_{\text {eff }}$ and $T$, and they become higher as $T$ increases when compared at the same $\phi_{\text {eff. }}$ The $T$-dependent modulus and viscosity result from a change in the strength of the hydrophobic nature of the PNIPAM chains, which governs the attractive interparticle interactions. The aggregations of the collapsed microgels considerably lower the threshold volume fraction of particle $\left(\phi_{\mathrm{c}}\right)$ for the formation of infinite percolation clusters compared with the values of $\phi_{\mathrm{c}}$ in the swollen state and for the random close packing of hard spheres.

\section{CONFLICT OF INTEREST}

The authors declare no conflict of interest.

1 Snowden, M. J., Chowdhry, B. Z., Vincent, B. \& Morris, G. E. Colloidal copolymer microgels of $\mathrm{N}$-isopropylacrylamide and acrylic acid: $\mathrm{pH}$, ionic strength and temperature effects. J. Chem. Soc. Faraday Transact. 92, 5013-5016 (1996).

2 Pelton, R. Temperature-sensitive aqueous microgels. Adv. Colloid Interface Sci. 85, 1-33 (2000).

3 Lyon, L. A., Debord, J. D., Debord, S. B., Jones, C. D., McGrath, J. G. \& Serpe, M. J. Microgel colloidal crystals. J. Phys. Chem. B 108, 19099-19108 (2004).

4 Lyon, L. A. \& Fernandez-Nieves, A. The polymer/colloid duality of microgel suspensions. Annu. Rev. Phys. Chem. 63, 25-43 (2011).

5 Richtering, W. Responsive emulsions stabilized by stimuli-sensitive microgels: emulsions with special non-pickering properties. Langmuir 28, 17218-17229 (2012).

6 Yunker, P. J., Chen, K., Gratale, M. D., Lohr, M. A., Still, T. \& Yodh, A. G. Physics in ordered and disordered colloidal matter composed of poly(N-isopropylacrylamide) microgel particles. Rep. Progress Phys. 77, 056601 (2014).

7 Cloitre, M. in Microgel Suspensions (eds Fernadez-Nieves A., Wyss H., Mattson J. \& Weitz D. A.) (Wiley-VCH, Weinheim, 2011).

8 Koumakis, N., Pamvouxoglou, A., Poulos, A. S. \& Petekidis, G. Direct comparison of the rheology of model hard and soft particle glasses. Soft Matter 8, 4271-4284 (2012).

9 Urayama, K., Saeki, T., Cong, S., Uratani, S., Takigawa, T., Murai, M. \& Suzuki, D. A simple feature of yielding behavior of highly dense suspensions of soft micro-hydrogel particles. Soft Matter 10, 9486-9495 (2014).

10 Malmsten, M. in Microgel Suspensions (eds Fernadez-Nieves A., Wyss H., Mattson J. \& Weitz D. A.) (Wiley-VCH, Weinheim, 2011).

11 Schild, H. G. Poly(N-isopropylacrylamide): experiment, theory and application. Progress Polym. Sci. 17, 163-249 (1992).

12 Pelton, R. H. \& Chibante, P. Preparation of aqueous latices with $\mathrm{N}$-isopropylacrylamide. Colloids Surfaces 20, 247-256 (1986).

13 Hirose, Y., Amiya, T., Hirokawa, Y. \& Tanaka, T. Phase transition of submicron gel beads. Macromolecules 20, 1342-1344 (1987).

14 Kiminta, O. D. M., Luckham, P. F. \& Lenon, S. The rheology of deformable and thermoresponsive microgel particles. Polymer 36, 4827-4831 (1995).

15 Senff, H. \& Richtering, W. Temperature sensitive microgel suspensions: Colloidal phase behavior and rheology of soft spheres. J. Chem. Phys. 111, 1705-1711 (1999).
16 Senff, H. \& Richtering, W. Influence of cross-link density on rheological properties of temperature-sensitive microgel suspensions. Colloid Polym. Sci. 278, 830-840 (2000).

17 Stieger, M., Pedersen, J. S., Lindner, P. \& Richtering, W. Are thermoresponsive microgels model systems for concentrated colloidal suspensions? A rheology and smallangle neutron scattering study. Langmuir 20, 7283-7292 (2004).

18 Tan, B. H., Pelton, R. H. \& Tam, K. C. Microstructure and rheological properties of thermo-responsive poly(N-isopropylacrylamide) microgels. Polymer 51, 3238-3243 (2010).

19 Romeo, G., Fernandez-Nieves, A., Wyss, H. M., Acierno, D. \& Weitz, D. A. Temperature-controlled transitions between glass, liquid, and gel states in dense p-NIPA suspensions. Adv. Mater. 22, 3441-3445 (2010).

20 Xun, W. \& Richtering, W. Dilution leading to viscosity increase based on the cononsolvency effect of temperature-sensitive microgel suspensions. Colloids Surfaces A Physicochem. Eng. Aspects 484, 377-385 (2015).

21 Zhou, Z., Jia, D., Hollingsworth, J. V., Cheng, H. \& Han, C. C. From repulsive to attractive glass: a rheological investigation. J. Chem. Phys. 143, 234901 (2015).

22 Wolthers, W., Van Den Ende, D., Breedveld, V., Duits, M. H. G., Potanin, A. A., Wientjes, R. H. W. \& Mellema, J. Linear viscoelastic behavior of aggregated colloidal dispersions. Phys. Rev. E 56, 5726-5733 (1997).

23 Yanez, J. A., Laarz, E. \& Bergstrom, L. Viscoelastic properties of particle gels. J. Colloid Interface Sci. 209, 162-172 (1999).

24 Mellema, M., Van Opheusden, J. H. J. \& Van Vliet, T. Categorization of rheological scaling models for particle gels applied to casein gels. J. Rheol. 46, 11-29 (2002).

25 Mohraz, A. \& Solomon, M. J. Orientation and rupture of fractal colloidal gels during start-up of steady shear flow. J. Rheol. 49, 657-681 (2005).

26 Dinsmore, A. D., Prasad, V., Wong, I. Y. \& Weitz, D. A. Microscopic structure and elasticity of weakly aggregated colloidal gels. Phys. Rev. Lett. 96, 185502 (2006).

27 Bouchoux, A., Debbou, B., Gesan-Guiziou, G., Famelart, M. H., Doublier, J. L. \& Cabane, B. Rheology and phase behavior of dense casein micelle dispersions. J. Chem. Phys. 131, 165106 (2009).

28 Romeo, G., Filippone, G., Russo, P. \& Acierno, D. Effects of particle dimension and matrix viscosity on the colloidal aggregation in weakly interacting polymer-nanoparticle composites: a linear viscoelastic analysis. Polym. Bull. 63, 883-895 (2009).

29 Varga, Z. \& Swan, J. W. Linear viscoelasticity of attractive colloidal dispersions. J. Rheol. 59, 1271-1298 (2015).

30 Trappe, V. \& Weitz, D. A. Scaling of the viscoelasticity of weakly attractive particles. Phys. Rev. Lett. 85, 449-452 (2000).

31 Suzuki, D. \& Horigome, K. Assembly of oppositely charged microgels at the air/water interface. J. Phys. Chem. B 117, 9073-9082 (2013).

32 Wolfe, M. S. \& Scopazzi, C. Rheology of swellable microgel dispersions - influence of crosslink density. J. Colloid Interface Sci. 133, 265-277 (1989).

33 Paulin, S. E., Ackerson, B. J. \& Wolfe, M. S. Equilibrium and shear induced nonequilibrium phase behavior of PMMA microgel spheres. J. Colloid Interface Sci. 178, 251-262 (1996).

34 Flickinger, G. L., Dairanieh, I. S. \& Zukoski, C. F. The rheology of aqueous polyurethane dispersions. J. Non-Newton Fluid 87, 283-305 (1999).

35 Borrega, R., Cloitre, M., Betremieux, I., Ernst, B. \& Leibler, L. Concentration dependence of the low-shear viscosity of polyelectrolyte micro-networks: From hard spheres to soft microgels. Europhys. Lett. 47, 729-735 (1999).

36 Kaneda, I., Sogabe, A. \& Nakajima, H. Water-swellable polyelectrolyte microgels polymerized in an inverse microemulsion using a nonionic surfactant. J. Colloid Interface Sci. 275, 450-457 (2004).

37 Omari, A., Tabary, R., Rousseau, D., Calderon, F. L., Monteil, J. \& Chauveteau, G. Soft water-soluble microgel dispersions: Structure and rheology. J. Colloid Interface Sci. 302, 537-546 (2006).

38 Ferry, J. D. Viscoelastic Properties of Polymers. Third edition (John Wiley \& Sons, New York, 1980).

39 Tam, K. C., Wu, X. Y. \& Pelton, R. H. Poly(N-isopropylacrylamide). II. Effect of polymer concentration, temperature, and surfactant on the viscosity of aqueous solutions. J. Polym. Sci. A 31, 963-969 (1993).

40 Badiger, M. V. \& Wolf, B. A. Shear induced demixing and rheological behavior of aqueous solutions of poly ( $\mathrm{N}$-isopropylacrylamide). Macromol. Chem. Phys. 204, 600-606 (2003).

41 Trappe, V., Prasad, V., Cipelletti, L., Segre, P. N. \& Weitz, D. A. Jamming phase diagram for attractive particles. Nature 411, 772-775 (2001).

42 Papir, Y. S. \& Krieger, I. M. Rheological studies on dispersions of uniform colloidal spheres. II. Dispersions in nonaqueous media. J. Colloid Interface Sci. 34, 126-130 (1970).

43 Van Der Werff, J. C. \& De Kruif, C. G. Hard-sphere colloidal dispersions: the scaling of rheological properties with particle size, volume fraction, and shear rate. J. Rheol. $\mathbf{3 3}$, 421-454 (1989).

44 Krieger, I. M. \& Dougherty, T. A mechanism for non-Newtonian flow in suspensions of rigid spheres. Trans. Soc. Rheol. 3, 137 (1959).

$45 \mathrm{Kratz}, \mathrm{K}$., Hellweg, T. \& Eimer, W. Influence of charge density on the swelling of colloidal poly(N-isopropylacrylamide-co-acrylic acid) microgels. Colloids Surfaces $A$ Physicochem. Eng. Aspects 170, 137-149 (2000). 